\title{
On a singular minimizing problem
}

\author{
Grey Ercole* and Gilberto de Assis Pereira \\ Departamento de Matemática - Universidade Federal de Minas Gerais \\ Belo Horizonte, MG, 30.123-970, Brazil. \\ grey@mat.ufmg.br, gilbertoapereira@yahoo.com.br
}

August 31, 2018

\begin{abstract}
For each $q \in(0,1)$ let$$
\lambda_{q}(\Omega):=\inf \left\{\|\nabla v\|_{L^{p}(\Omega)}^{p}: v \in W_{0}^{1, p}(\Omega) \text { and } \int_{\Omega}|v|^{q} \mathrm{~d} x=1\right\},
$$

where $p>1$ and $\Omega$ is a bounded and smooth domain of $\mathbb{R}^{N}, N \geq 2$. We first show that

$$
0<\mu(\Omega):=\lim _{q \rightarrow 0^{+}} \lambda_{q}(\Omega)|\Omega|^{\frac{p}{q}}<\infty
$$
\end{abstract}

where $|\Omega|=\int_{\Omega} \mathrm{d} x$. Then, we prove that

$$
\mu(\Omega)=\min \left\{\|\nabla v\|_{L^{p}(\Omega)}^{p}: v \in W_{0}^{1, p}(\Omega) \text { and } \lim _{q \rightarrow 0^{+}}\left(\frac{1}{|\Omega|} \int_{\Omega}|v|^{q} \mathrm{~d} x\right)^{\frac{1}{q}}=1\right\}
$$

and that $\mu(\Omega)$ is reached by a function $u \in W_{0}^{1, p}(\Omega)$, which is positive in $\Omega$, belongs to $C^{0, \alpha}(\bar{\Omega})$, for some $\alpha \in(0,1)$, and satisfies

$$
-\operatorname{div}\left(|\nabla u|^{p-2} \nabla u\right)=\mu(\Omega)|\Omega|^{-1} u^{-1} \quad \text { in } \quad \Omega, \quad \text { and } \quad \int_{\Omega} \log u \mathrm{~d} x=0 .
$$

We also show that $\mu(\Omega)^{-1}$ is the best constant $C$ in the following log-Sobolev type inequality

$$
\exp \left(\frac{1}{|\Omega|} \int_{\Omega} \log |v|^{p} \mathrm{~d} x\right) \leq C\|\nabla v\|_{L^{p}(\Omega)}^{p}, \quad v \in W_{0}^{1, p}(\Omega)
$$

and that this inequality becomes an equality if, and only if, $v$ is a scalar multiple of $u$ and $C=\mu(\Omega)^{-1}$.

2010 Mathematics Subject Classification. 35B40; 35J25; 35J92.

Keywords: Asymptotic behavior, log-Sobolev inequality, p-Laplacian, singular problem.

\section{Introduction}

Let $p>1$ be fixed and let $\Omega \subset \mathbb{R}^{N}, N \geq 2$, be a bounded and smooth domain. For each $q \in(0,1)$ let us define

$$
\lambda_{q}(\Omega):=\inf \left\{\|\nabla v\|_{p}^{p}: v \in W_{0}^{1, p}(\Omega) \text { and } \int_{\Omega}|v|^{q} \mathrm{~d} x=1\right\}
$$

where $\|\cdot\|_{s}$ denotes the standard norm of the Lebesgue space $L^{s}(\Omega), 1 \leq s \leq \infty$.

\footnotetext{
${ }^{*}$ Corresponding author
} 
As proved in [1], $\lambda_{q}(\Omega)$ is achieved by a positive function $u_{q} \in W_{0}^{1, p}(\Omega) \cap C^{1}(\Omega)$ satisfying the singular Dirichlet problem

$$
\begin{cases}-\Delta_{p} v=\lambda_{q}(\Omega)|v|^{q-2} v & \text { in } \Omega \\ v=0 & \text { on } \partial \Omega,\end{cases}
$$

in the weak sense, where $\Delta_{p} v=\operatorname{div}\left(|\nabla v|^{p-2} \nabla v\right)$ is the $p$-Laplacian operator. Moreover, it follows from [10, Theorem 1.1 (i)] that $u_{q} \in C^{1, \alpha}(\bar{\Omega})$, for some $\alpha \in(0,1)$.

In this paper we first show that

$$
0<\mu(\Omega):=\lim _{q \rightarrow 0^{+}} \lambda_{q}(\Omega)|\Omega|^{\frac{p}{q}}<\infty,
$$

where $|D|$ stands for the $N$-dimensional Lebesgue volume of $D \subset \mathbb{R}^{N}$, i. e. $|D|=\int_{D} \mathrm{~d} x$.

Then, we prove that

$$
\mu(\Omega)=\min \left\{\|\nabla v\|_{p}^{p}: v \in W_{0}^{1, p}(\Omega) \text { and } \lim _{q \rightarrow 0^{+}}\left(\frac{1}{|\Omega|} \int_{\Omega}|v|^{q} \mathrm{~d} x\right)^{\frac{1}{q}}=1\right\}
$$

and that the minimum is reached by a function $u \in W_{0}^{1, p}(\Omega)$, which is positive in $\Omega$, belongs to $C^{0, \alpha}(\bar{\Omega})$, for some $\alpha \in(0,1)$, and satisfies:

(i) $u=\lim _{q \rightarrow 0^{+}}|\Omega|^{\frac{1}{q}} u_{q}$ in $W_{0}^{1, p}(\Omega)$;

(ii) $-\Delta_{p} u=\mu(\Omega)|\Omega|^{-1} u^{-1}$ in $\Omega$; and

(iii) $\int_{\Omega} \log u \mathrm{~d} x=0$.

Exploring (4) we also prove that $\mu(\Omega)^{-1}$ is the best constant $C$ in the following log-Sobolev type inequality

$$
\exp \left(\frac{1}{|\Omega|} \int_{\Omega} \log |v|^{p} \mathrm{~d} x\right) \leq C\|\nabla v\|_{p}^{p}, \quad v \in W_{0}^{1, p}(\Omega),
$$

and that $\mu(\Omega)^{-1}$ is reached if, and only if, $v$ is a scalar multiple of $u$, which is the unique case where the inequality becomes an equality. Up to our knowledge, these facts are entirely new.

It is easy to check that for each fixed $\lambda>0$ the function $u_{\lambda}:=\left(\frac{\lambda|\Omega|}{\mu(\Omega)}\right)^{\frac{1}{p}} u$ is a positive weak solution of the singular problem

$$
\begin{cases}-\Delta_{p} v=\lambda v^{-1} & \text { in } \Omega \\ v=0 & \text { on } \partial \Omega .\end{cases}
$$

The function $u_{\lambda}$ is, in fact, the unique positive solution of (5). This uniqueness result follows from a simple and well-known inequality involving vectors of $\mathbb{R}^{N}$. Existence and regularity of weak solutions for (5) were first studied in the particular case $p=2$ (see [4, 13, 16), whereas the case $p>1$ has received more attention in the last decade (see [3, 9, 10, 14, and references therein).

We remark that the differentiability of the functional $v \in W_{0}^{1, p}(\Omega) \mapsto \lambda \int_{\Omega} \log |v| \mathrm{d} x \in[-\infty, \infty)$ is a delicate question, which makes it difficult to apply variational methods to obtain the positive solution of (5). Thus, $u_{\lambda}$ has generally been obtained by nonvariational methods, mainly the sub-super solution method. As for regularity, it is proved in [10, Theorem 2.2 (ii)] that $u_{\lambda} \in C^{0, \alpha}(\bar{\Omega})$, for some $\alpha \in(0,1)$.

We emphasize that besides providing a new existence proof of $u_{\lambda}$, we show that

$$
\int_{\Omega} \log u_{\lambda} \mathrm{d} x=\frac{|\Omega|}{p} \log \left(\frac{\lambda|\Omega|}{\mu(\Omega)}\right) \in(-\infty, \infty)
$$


This property of $u_{\lambda}$ was not known up to now. It comes from the connection between (5) and the minimizing problem (4).

Also in this paper, we show that the formal energy functional associated with (5),

$$
J_{\lambda}(v):= \begin{cases}\frac{1}{p} \int_{\Omega}|\nabla v|^{p} \mathrm{~d} x-\lambda \int_{\Omega} \log |v| \mathrm{d} x, & \text { if } \int_{\Omega} \log |v| \mathrm{d} x \in(-\infty, \infty) \\ \infty, & \text { if } \int_{\Omega} \log |v| \mathrm{d} x=-\infty\end{cases}
$$

attains its minimum value $\frac{\lambda|\Omega|}{p}\left(1-\log \left(\frac{\lambda|\Omega|}{\mu(\Omega)}\right)\right)$ only at the functions $u_{\lambda}$ and $-u_{\lambda}$.

We end the paper by describing the asymptotic behavior of the pair $\left(\lambda_{q}(\Omega),\left\|u_{q}\right\|_{\infty}\right)$, as $q \rightarrow 0^{+}$. That is, we determine when these quantities either go to 0 or to $\infty$ or remain bounded from above and from below, when $q \rightarrow 0^{+}$. More precisely, we obtain directly from (3) that

$$
\lim _{q \rightarrow 0^{+}} \lambda_{q}(\Omega)= \begin{cases}\infty & \text { if }|\Omega|<1 \\ \mu(\Omega) & \text { if }|\Omega|=1 \\ 0 & \text { if }|\Omega|>1,\end{cases}
$$

and apply lower and upper estimates (derived in Section 2) to show that

$$
\lim _{q \rightarrow 0^{+}}\left\|u_{q}\right\|_{\infty}=\left\{\begin{array}{lll}
\infty & \text { if } & |\Omega|<1 \\
0 & \text { if } & |\Omega|>1
\end{array}\right.
$$

and that

$$
0<A \mu(\Omega)^{\frac{1}{p}} \leq \lim _{q \rightarrow 0^{+}}\left\|u_{q}\right\|_{\infty} \leq B \mu(\Omega)^{\frac{1}{p}}, \quad \text { if }|\Omega|=1,
$$

where $A$ and $B$ are positive constants that depend only on $N$ and $p$.

The result in (6) for the case $|\Omega|<1$ has recently been obtained in (1). The cases $|\Omega| \geq 1$ in (6) as well as (7) and (8) are new observations.

Thus, (6), (7) and (8) provide complementary information on how the function $q \in\left(0, p^{\star}\right) \mapsto\left(\lambda_{q}(\Omega),\left\|u_{q}\right\|_{\infty}\right) \in$ $\mathbb{R}^{2}$ behaves at the endpoints of its domain. In fact, the behavior of this function as $q \rightarrow p^{\star}$ is well known:

$$
\lim _{q \rightarrow p^{\star}} \lambda_{q}(\Omega)=\left\{\begin{array}{lll}
S_{N, p}, & \text { if } & 1<p<N \\
0, & \text { if } & p=N>1 \\
\Lambda_{p}(\Omega), & \text { if } & p>N
\end{array}\right.
$$

and

$$
\lim _{q \rightarrow p^{\star}}\left\|u_{q}\right\|_{\infty}=\left\{\begin{array}{lll}
\infty, & \text { if } 1<p<N \\
C_{N}, & \text { if } p=N>1 \\
1, & \text { if } \quad p>N
\end{array}\right.
$$

where

$$
\Lambda_{p}(\Omega):=\min \left\{\|\nabla u\|_{p}^{p}: u \in W_{0}^{1, p}(\Omega) \text { and }\|u\|_{\infty}=1\right\},
$$

$S_{N, p}$ is the well-known Sobolev constant, defined by

$$
S_{N, p}:=\pi^{\frac{p}{2}} N\left(\frac{N-p}{p-1}\right)^{p-1}\left(\frac{\Gamma(N / p) \Gamma(1+N-N / p)}{\Gamma(1+N / 2) \Gamma(N)}\right)^{\frac{p}{N}}
$$

$\Gamma$ denoting the Gamma Function, and $C_{N}$ is a positive constant that does not depend on $\Omega$.

For (9) and [10) we refer to [1, 5], [15] and [7, respectively to the cases $1\langle p\langle N, p=N\rangle 1$ and $p\rangle N\rangle 1$. 


\section{Preliminaries}

In this section, we present some properties of the weak solutions of the singular Dirichlet problem

$$
\begin{cases}-\Delta_{p} v=\lambda v^{q-1} & \text { in } \Omega, \quad 0 \leq q<1, \quad \lambda>0 \\ v>0 & \text { in } \Omega, \\ v=0 & \text { on } \partial \Omega\end{cases}
$$

which will be used in the paper. A weak solution of (12) is a function $v \in W_{0}^{1, p}(\Omega)$ such that $\operatorname{essinf}_{K} v>0$ in each compact $K \subset \Omega$ and

$$
\int_{\Omega}|\nabla v|^{p-2} \nabla v \cdot \nabla \varphi \mathrm{d} x=\lambda \int_{\Omega} v^{q-1} \varphi \mathrm{d} x, \text { for all } \varphi \in W_{0}^{1, p}(\Omega) .
$$

Next, we present a simple uniqueness proof for (12), which makes use of the following well-known inequality:

$$
\left(|x|^{p-2} x-|y|^{p-2} y\right) \cdot(x-y) \geq 0 \text { for all } x, y \in \mathbb{R}^{N} .
$$

Proposition 1 Let $u_{1}, u_{2} \in W_{0}^{1, p}(\Omega)$ be weak solutions of (12). Then, $u_{2}=u_{1}$ a. e. in $\Omega$.

Proof. Taking $\varphi=u_{2}-u_{1}$ in (13) we obtain

$$
\int_{\Omega}\left(\left|\nabla u_{2}\right|^{p-2} \nabla u_{2}-\left|\nabla u_{1}\right|^{p-2} \nabla u_{1}\right) \cdot \nabla\left(u_{2}-u_{1}\right) \mathrm{d} x=\lambda \int_{\Omega}\left(u_{2}^{q-1}-u_{1}^{q-1}\right)\left(u_{2}-u_{1}\right) \mathrm{d} x .
$$

It follows from (14) that the integrand in the left-hand side of (15) is nonnegative. It is easy to see that the integrand of the right-hand side of (15) cannot be positive. Thus, both of them must be null almost everywhere in $\Omega$, which implies that $u_{2}=u_{1}$ a.e. in $\Omega$.

In the sequel we derive estimates for the weak solutions of (12) depending explicitly on $q \in[0,1)$.

Let us recall that

$$
\lambda_{q}\left(D^{*}\right) \leq \lambda_{q}(D), \quad 0<q<p^{\star}
$$

where $D$ is a general bounded and smooth domain of $\mathbb{R}^{N}$ and $D^{*}$ is the ball centered at the origin and with the same volume as $D$, that is, $\left|D^{*}\right|=|D|$.

Inequality (16) comes from well known properties of Schwarz symmetrization (see [1]) and, among other important utilities, it provides a lower bound for $\lambda_{q}(D)$ in terms of $|D|$ and $\lambda_{q}\left(B_{1}\right)$, where $B_{1}$ denotes the unit ball of $\mathbb{R}^{N}$. In fact, one can show that

$$
\lambda_{q}\left(D^{*}\right)=\lambda_{q}\left(B_{1}\right)\left(\frac{\left|D^{*}\right|}{\omega_{N}}\right)^{1-\frac{p}{N}-\frac{p}{q}}, \quad 0<q<p^{\star}
$$

where $\omega_{N}=\left|B_{1}\right|$.

Hence, by combining (16) and (17) one obtains the following version of the well-known Poincaré-Sobolev inequality

$$
\left(\int_{D}|v|^{q} \mathrm{~d} x\right)^{\frac{p}{q}} \leq \frac{|D|^{\frac{p}{q}+\frac{p}{N}-1}}{\lambda_{q}\left(B_{1}\right)\left|B_{1}\right|^{\frac{p}{q}+\frac{p}{N}-1}}\|\nabla v\|_{L^{p}(D)}^{p} \text {, for all } v \in W_{0}^{1, p}(D) \text { and } 0<q<p^{\star} .
$$

When $q=p$ we have

$$
\int_{D}|v|^{p} \mathrm{~d} x \leq \frac{|D|^{\frac{p}{N}}}{C_{N, p}} \int_{D}|\nabla v|^{p} \mathrm{~d} x, \text { for all } v \in W_{0}^{1, p}(D),
$$

where $C_{N, p}=\lambda_{p}\left(B_{1}\right)\left|B_{1}\right|^{\frac{p}{N}}$, a positive constant that depends only on $p$ and $N$.

The following lemma is an adaptation of [6, Theorem 4.1] which, in its turn, is based on classical set level techniques (see [2, 12]). 
Lemma 2 If $u \in W_{0}^{1, p}(\Omega)$ is a weak solution of (12), then $u \in L^{\infty}(\Omega)$ and

$$
\|u\|_{\infty} \leq K_{N, p}|\Omega|^{\frac{p}{N(p-q)}} \lambda^{\frac{1}{p-q}}
$$

where $K_{N, p}$ is a positive constant depending only on $N$ and $p$.

Proof. For each $t>0$, let

$$
E_{t}:=\{x \in \Omega: u>t\} \text { and }(u-t)_{+}:=\max \{u-t, 0\} \in W_{0}^{1, p}(\Omega) .
$$

Let us suppose $\left|E_{t}\right|>0$. Since

$$
\int_{\Omega}|\nabla u|^{p-2} \nabla u \cdot \nabla(u-t)_{+} \mathrm{d} x=\int_{E_{t}}|\nabla u|^{p} \mathrm{~d} x
$$

and

$$
\int_{\Omega} u^{q-1}(u-t)_{+} \mathrm{d} x=\int_{E_{t}} u^{q-1}(u-t) \mathrm{d} x \leq t^{q-1} \int_{E_{t}}(u-t) \mathrm{d} x,
$$

(note that $q-1<0$ ) we obtain from (13) that

$$
\int_{E_{t}}|\nabla u|^{p} \mathrm{~d} x \leq \lambda t^{q-1} \int_{E_{t}}(u-t) \mathrm{d} x .
$$

Now, we estimate $\int_{E_{t}}|\nabla u|^{p} \mathrm{~d} x$ from below. For this, we apply Hölder inequality and the estimate (18) with $D=E_{t}$ to obtain

$$
\left(\int_{E_{t}}(u-t) \mathrm{d} x\right)^{p} \leq\left|E_{t}\right|^{p-1} \int_{E_{t}}(u-t)^{p} \mathrm{~d} x \leq \frac{\left|E_{t}\right|^{p-1}\left|E_{t}\right|^{\frac{p}{N}}}{C_{N, p}} \int_{E_{t}}|\nabla u|^{p} \mathrm{~d} x .
$$

Hence,

$$
C_{N, p}\left|E_{t}\right|^{-\frac{p}{N}+1-p}\left(\int_{E_{t}}(u-t) \mathrm{d} x\right)^{p} \leq \int_{E_{t}}|\nabla u|^{p} \mathrm{~d} x
$$

and, by taking into account (20), we get

$$
C_{N, p}\left|E_{t}\right|^{-\frac{p}{N}+1-p}\left(\int_{E_{t}}(u-t) \mathrm{d} x\right)^{p} \leq \lambda t^{q-1} \int_{E_{t}}(u-t) \mathrm{d} x
$$

which is equivalent to

$$
\left(\int_{E_{t}}(u-t) \mathrm{d} x\right)^{p-1} \leq \frac{\lambda}{C_{N, p}} t^{q-1}\left|E_{t}\right|^{\frac{p+N(p-1)}{N}} .
$$

This latter inequality can be rewritten as

$$
\left(\int_{E_{t}}(u-t) \mathrm{d} x\right)^{\frac{N(p-1)}{p+N(p-1)}} \leq\left(\frac{\lambda}{C_{N, p}} t^{q-1}\right)^{\frac{N}{p+N(p-1)}}\left|E_{t}\right| .
$$

Let us define

$$
f(t):=\int_{E_{t}}(u-t) \mathrm{d} x=\int_{t}^{\infty}\left|E_{s}\right| \mathrm{d} s,
$$

where the second equality follows from Cavalieri's principle.

Since $f^{\prime}(t)=-\left|E_{t}\right|$ the inequality in (21) can be rewritten as

$$
t^{\frac{(1-q) N}{p+N(p-1)}} \leq-\left(\frac{\lambda}{C_{N, p}}\right)^{\frac{N}{p+N(p-1)}} f(t)^{-\frac{N(p-1)}{p+N(p-1)}} f^{\prime}(t) .
$$


Integration of (22) yields

$$
\begin{aligned}
\frac{p+N(p-1)}{p+N(p-q)} t^{\frac{p+N(p-q)}{p+N(p-1)}} & \leq\left(\frac{\lambda}{C_{N, p}}\right)^{\frac{N}{p+N(p-1)}} \frac{p+N(p-1)}{p}\left[f(0)^{\frac{p}{p+N(p-1)}}-f(t)^{\frac{p}{p+N(p-1)}}\right] \\
& \leq\left(\frac{\lambda}{C_{N, p}}\right)^{\frac{N}{p+N(p-1)}} \frac{p+N(p-1)}{p}\left(\|u\|_{1}\right)^{\frac{p}{p+N(p-1)}}<\infty .
\end{aligned}
$$

We have concluded that if $\left|E_{t}\right|>0$ then $t \leq K$, where $K$ is a positive constant that does not depend on $t$. Of course, this implies that $\|u\|_{\infty}<\infty$.

Hence, we have

$$
\begin{aligned}
\frac{p+N(p-1)}{p+N(p-q)} t^{\frac{p+N(p-q)}{p+N(p-1)}} & \leq\left(\frac{\lambda}{C_{N, p}}\right)^{\frac{N}{p+N(p-1)}} \frac{p+N(p-1)}{p}\left(\|u\|_{1}\right)^{\frac{p}{p+N(p-1)}} \\
& \leq\left(\frac{\lambda}{C_{N, p}}\right)^{\frac{N}{p+N(p-1)}} \frac{p+N(p-1)}{p}\left(\|u\|_{\infty}|\Omega|\right)^{\frac{p}{p+N(p-1)}}
\end{aligned}
$$

and then, after making $t \rightarrow\|u\|_{\infty}$, we obtain

$$
\|u\|_{\infty} \leq\left(\frac{\lambda}{C_{N, p}}\right)^{\frac{1}{p-q}}\left(\frac{p+N(p-q)}{p}\right)^{\frac{p+N(p-1)}{N(p-q)}}|\Omega|^{\frac{p}{N(p-q)}}
$$

which leads to (19) with

$$
K_{N, p}:=\sup _{0 \leq q \leq 1} C_{N, p}^{-\frac{1}{p-q}}\left(\frac{p+N(p-q)}{p}\right)^{\frac{p+N(p-1)}{N(p-q)}} .
$$

In the next lemma, $\phi_{p} \in W_{0}^{1, p}(\Omega)$ denotes the $p$-torsion function of $\Omega$, that is, the weak solution of the $p$-torsional creep problem

$$
\begin{cases}-\Delta_{p} v=1 & \text { in } \Omega \\ v=0 & \text { on } \partial \Omega\end{cases}
$$

It is well known that the function $\phi_{p}$ is positive in $\Omega$ and belongs to $C^{1, \alpha}(\bar{\Omega})$, for some $\alpha \in(0,1)$.

Lemma 3 If $u \in W_{0}^{1, p}(\Omega)$ is a weak solution of (12), then

$$
0<\left(K_{N, p}|\Omega|^{\frac{p}{N(p-q)}}\right)^{\frac{q-1}{p-1}} \lambda^{\frac{1}{p-q}} \phi_{p}(x) \leq u(x), \quad \text { for almost every } x \in \Omega .
$$

Proof. Let $0 \leq \varphi \in W_{0}^{1, p}(\Omega)$ and $c:=\left(\lambda\|u\|_{\infty}^{q-1}\right)^{\frac{1}{p-1}}$. Since

$$
\begin{aligned}
\int_{\Omega}|\nabla u|^{p-2} \nabla u \cdot \nabla \varphi \mathrm{d} x & =\lambda \int_{\Omega} u^{q-1} \varphi \mathrm{d} x \\
& \geq \lambda\|u\|_{\infty}^{q-1} \int_{\Omega} \varphi \mathrm{d} x \\
& =\int_{\Omega} c^{p-1} \varphi \mathrm{d} x=\int_{\Omega}\left|\nabla\left(c \phi_{p}\right)\right|^{p-2} \nabla\left(c \phi_{p}\right) \cdot \nabla \varphi \mathrm{d} x,
\end{aligned}
$$

the weak comparison principle guarantees that

$$
c \phi_{p}(x) \leq u(x), \quad \text { for almost every } x \in \Omega .
$$

This leads to (23) since (19) implies that

$$
c \geq \lambda^{\frac{1}{p-1}}\left(K_{N, p}|\Omega|^{\frac{p}{N(p-q)}} \lambda^{\frac{1}{p-q}}\right)^{\frac{q-1}{p-1}}=\left(K_{N, p}|\Omega|^{\frac{p}{N(p-q)}}\right)^{\frac{q-1}{p-1}} \lambda^{\frac{1}{p-q}}, \quad \text { for almost every } x \in \Omega .
$$


Remark 4 It follows from Lemma 圆 and Lemma 3 that if $u \in W_{0}^{1, p}(\Omega)$ is a weak solution of (12) then

$$
0<A \lambda^{\frac{p-1}{p-q}} \leq \lambda u(x)^{q-1} \leq B \lambda^{\frac{p-1}{p-q}} \phi_{p}(x)^{q-1}, \quad \text { for almost every } x \in \Omega,
$$

where $A$ and $B$ are positive constants that depend only on $N, p$ and $|\Omega|$. This fact implies that if $\Omega^{\prime}$ is a subdomain of $\Omega$ such that $\overline{\Omega^{\prime}} \subset \Omega$, then $\lambda u^{q-1}$ is bounded in $\overline{\Omega^{\prime}}$.

\section{The main results}

One can check, as a simple application of the Hölder inequality, that for each $v \in L^{1}(\Omega)$ the function $q \in(0,1] \mapsto$ $\left(\frac{1}{|\Omega|} \int_{\Omega}|v|^{q} \mathrm{~d} x\right)^{\frac{1}{q}}$ is increasing. This fact has two immediate consequences: it implies that

$$
0 \leq \lim _{q \rightarrow 0^{+}}\left(\frac{1}{|\Omega|} \int_{\Omega}|v|^{q} \mathrm{~d} x\right)^{\frac{1}{q}}=\inf _{0<s \leq 1}\left(\frac{1}{|\Omega|} \int_{\Omega}|v|^{s} \mathrm{~d} x\right)^{\frac{1}{s}} \leq \frac{\|v\|_{1}}{|\Omega|}, \quad v \in L^{1}(\Omega)
$$

and also that the function $q \in(0,1] \mapsto \lambda_{q}(\Omega)|\Omega|^{\frac{p}{q}}$ is decreasing, so that we can define

$$
\mu(\Omega):=\lim _{q \rightarrow 0^{+}} \lambda_{q}(\Omega)|\Omega|^{\frac{p}{q}}=\sup _{0<s \leq 1} \lambda_{s}(\Omega)|\Omega|^{\frac{p}{s}} .
$$

Of course,

$$
0<\lambda_{1}(\Omega)|\Omega|^{p} \leq \mu(\Omega) \leq \infty
$$

Our first goal in this section is to show that $\mu(\Omega)<\infty$.

Lemma 5 One has

$$
\lim _{q \rightarrow 0^{+}}\left(\int_{0}^{1}\left(1-t^{\frac{1}{q}}\right)^{N} \mathrm{~d} t\right)^{\frac{1}{q}}=e^{-1-\frac{1}{2}-\frac{1}{3}-\cdots-\frac{1}{N}}, \quad N \geq 2 .
$$

Proof. We have

$$
\lim _{q \rightarrow 0^{+}}\left(\int_{0}^{1}\left(1-t^{\frac{1}{q}}\right)^{N} \mathrm{~d} t\right)^{\frac{1}{q}}=\lim _{s \rightarrow \infty}\left(\int_{0}^{1}\left(1-t^{s}\right)^{N} \mathrm{~d} t\right)^{s}=e^{L}
$$

where

$$
\begin{aligned}
L & :=\lim _{s \rightarrow \infty} \frac{\ln \left(\int_{0}^{1}\left(1-t^{s}\right)^{N} \mathrm{~d} t\right)}{s^{-1}} \\
& =\lim _{s \rightarrow \infty} \frac{s^{2} N \int_{0}^{1}\left(1-t^{s}\right)^{N-1} t^{s} \ln t \mathrm{~d} t}{\int_{0}^{1}\left(1-t^{s}\right)^{N} \mathrm{~d} t}=\lim _{s \rightarrow \infty} s^{2} N \int_{0}^{1}\left(1-t^{s}\right)^{N-1} t^{s} \ln t \mathrm{~d} t .
\end{aligned}
$$

After making the change of variable $\tau=t^{s}$ in the latter integral, we obtain

$$
L=N \lim _{s \rightarrow \infty} \int_{0}^{1}(1-\tau)^{N-1} \tau^{\frac{1}{s}} \ln \tau \mathrm{d} \tau=N \int_{0}^{1}(1-\tau)^{N-1} \ln \tau \mathrm{d} \tau .
$$

In order to finish the proof, it is enough to verify that

$$
N \int_{0}^{1}(1-\tau)^{N-1} \ln \tau \mathrm{d} \tau=-1-\frac{1}{2}-\frac{1}{3}-\cdots-\frac{1}{N}, \quad N \geq 2 .
$$

For this, let $I(N):=N \int_{0}^{1}(1-\tau)^{N-1} \ln \tau \mathrm{d} \tau$. After some simple calculations one can show that

$$
I(N+1)=I(N)-\frac{1}{N+1}, \quad N \geq 2
$$

It is easy to check that $I(2)=-1-\frac{1}{2}$. Hence, by using the recursive formula (26), we arrive at (25). 
Lemma 6 Suppose that $\Omega$ is a bounded domain, star-shaped with respect to $x_{0} \in \mathbb{R}^{N}$. There exists $\rho \in C(\bar{\Omega})$ such that: $0<\rho \leq 1$ in $\Omega, \rho\left(x_{0}\right)=1, \rho=0$ on $\partial \Omega$ and

$$
\lim _{q \rightarrow 0^{+}}\left(\frac{1}{|\Omega|} \int_{\Omega}|\rho|^{q} \mathrm{~d} x\right)^{\frac{1}{q}}=e^{-1-\frac{1}{2}-\frac{1}{3}-\cdots-\frac{1}{N}}
$$

In particular, any function $v \in W_{0}^{1, p}(\Omega)$ such that $v \geq \rho$ in $\Omega$ satisfies

$$
\lim _{q \rightarrow 0^{+}}\left(\frac{1}{|\Omega|} \int_{\Omega}|v|^{q} \mathrm{~d} x\right)^{\frac{1}{q}} \geq e^{-1-\frac{1}{2}-\frac{1}{3}-\cdots-\frac{1}{N}}>0 .
$$

Proof. We will assume in this proof, without loss of generality, that $x_{0}=0$.

For each $0 \neq x \in \bar{\Omega}$, let $r(x)$ be the unique positive number such that

$$
r(x) x \in \partial \Omega .
$$

Of course, $r(x) \geq 1$ and $r(x) \rightarrow \infty$ as $x \rightarrow 0$. Moreover, if $x \in \Omega$ and $\alpha>0$ is such that $\alpha x \in \Omega$, then $r(\alpha x) \alpha x=r(x) x$, so that

$$
r(\alpha x)=\frac{r(x)}{\alpha}
$$

Let us define $\rho: \bar{\Omega} \mapsto[0,1]$ by

$$
\rho(x):=\left\{\begin{array}{lll}
1-\frac{1}{r(x)} & \text { if } & x \in \bar{\Omega} \quad, \quad x \neq 0 . \\
1 & \text { if } \quad x=0 .
\end{array}\right.
$$

The graph of $\rho$ in $\mathbb{R}^{N} \times \mathbb{R}$ is the cone of base $\Omega$, height 1 and vertex at the point $(0,1) \in \mathbb{R}^{N} \times \mathbb{R}$.

For each $t \in[0,1)$ the change of variable $x=(1-t) y$ yields

$$
|\{\rho(x)>t\}|=\int_{\{\rho(x)>t\}} \mathrm{d} x=\int_{\{\rho(y)>0\}}(1-t)^{N} \mathrm{~d} y=(1-t)^{N}|\Omega| .
$$

Indeed, by taking $\alpha=(1-t)$ one has

$$
\rho(\alpha y)=1-\frac{1}{r(\alpha y)}=1-\frac{\alpha}{r(y)}=1-\alpha+\alpha\left(1-\frac{1}{r(y)}\right)=1-\alpha+\alpha \rho(y) .
$$

It follows that

$$
t<\rho((1-t) y)=t+(1-t) \rho(y) \Longleftrightarrow 0<\rho(y) .
$$

Thus, (27) and Cavalieri's principle yield

$$
\int_{\Omega} \rho^{q} \mathrm{~d} x=\int_{0}^{1}\left|\left\{\rho(x)^{q}>t\right\}\right| \mathrm{d} t=\int_{0}^{1}\left|\left\{\rho(x)>t^{\frac{1}{q}}\right\}\right| \mathrm{d} t=\int_{0}^{1}\left(1-t^{\frac{1}{q}}\right)^{N}|\Omega| \mathrm{d} t,
$$

so that

$$
\lim _{q \rightarrow 0^{+}}\left(\frac{1}{|\Omega|} \int_{\Omega} \rho^{q} \mathrm{~d} x\right)^{\frac{1}{q}}=\lim _{q \rightarrow 0^{+}}\left(\int_{0}^{1}\left(1-t^{\frac{1}{q}}\right)^{N} \mathrm{~d} t\right)^{\frac{1}{q}}=e^{-1-\frac{1}{2}-\frac{1}{3}-\cdots-\frac{1}{N}} .
$$

Remark 7 If $\Omega=B_{R}$ is the ball centered at the origin with radius $R$, then $\rho(x)=1-\frac{|x|}{R}$ and

$$
\frac{1}{\left|B_{R}\right|} \int_{B_{R}} \rho^{q} \mathrm{~d} x=\int_{0}^{1}\left(1-t^{\frac{1}{q}}\right)^{N} \mathrm{~d} t
$$


In the proof of the following theorem we will write $\Omega$ as a finite union of star-shaped subdomains. This decomposition is quite general in the sense that it is valid for bounded domains with low regularity as, for instance, those with Lipschitz boundary (see [8, Lemma II.1.3]).

Theorem 8 There exists $v \in W_{0}^{1, p}(\Omega)$ such that $v>0$ in $\Omega$ and

$$
\lim _{q \rightarrow 0^{+}}\left(\frac{1}{|\Omega|} \int_{\Omega}|v|^{q} \mathrm{~d} x\right)^{\frac{1}{q}}=1
$$

Moreover,

$$
\mu(\Omega) \leq\|\nabla v\|_{p}^{p}
$$

Proof. Let $\Omega_{1}, \Omega_{2}, \ldots, \Omega_{m}$ be star-shaped subdomains of $\Omega$ such that $\Omega=\bigcup_{j=1}^{m} \Omega_{j}$ (not necessarily disjoint).

According Lemma 6, for each $j \in \Lambda:=\{1,2, \ldots, m\}$ we can take $v_{j} \in W_{0}^{1, p}\left(\Omega_{j}\right)$ such that $v_{j}>0$ in $\Omega_{j}$ and

$$
e^{-1-\frac{1}{2}-\frac{1}{3}-\cdots-\frac{1}{N}} \leq \lim _{q \rightarrow 0^{+}}\left(\frac{1}{\left|\Omega_{j}\right|} \int_{\Omega_{j}}\left|v_{j}\right|^{q} \mathrm{~d} x\right)^{\frac{1}{q}}=\inf _{0<s \leq 1}\left(\frac{1}{\left|\Omega_{j}\right|} \int_{\Omega_{j}}\left|v_{j}\right|^{s} \mathrm{~d} x\right)^{\frac{1}{s}} .
$$

Thus,

$$
e^{-1-\frac{1}{2}-\frac{1}{3}-\cdots-\frac{1}{N}} \leq\left(\frac{1}{\left|\Omega_{j}\right|} \int_{\Omega_{j}}\left|v_{j}\right|^{q} \mathrm{~d} x\right)^{\frac{1}{q}}, \quad j \in \Lambda, \quad 0<q \leq 1 .
$$

By extending $v_{j}$ to zero outside $\Omega_{j}$ we can consider that $v_{j}$ belongs to $W_{0}^{1, p}(\Omega)$. Thus,

$$
V:=\sum_{j=1}^{m} v_{j} \in W_{0}^{1, p}(\Omega)
$$

Now, let $q_{n} \rightarrow 0^{+}$and, for each $n \in \mathbb{N}$, let $j_{n} \in \Lambda$ be such that

$$
\frac{1}{\left|\Omega_{j_{n}}\right|} \int_{\Omega_{j_{n}}}\left|v_{j_{n}}\right|^{q_{n}} \mathrm{~d} x=\min \left\{\frac{1}{\left|\Omega_{j}\right|} \int_{\Omega_{j}}\left|v_{j}\right|^{q_{n}} \mathrm{~d} x: j \in \Lambda\right\} .
$$

Then, for each fixed $n \in \mathbb{N}$ we have

$$
\begin{aligned}
\int_{\Omega}|V|^{q_{n}} \mathrm{~d} x & =\sum_{j=1}^{m}\left(\frac{1}{\left|\Omega_{j}\right|} \int_{\Omega_{j}}\left|v_{j}\right|^{q_{n}} \mathrm{~d} x\right)\left|\Omega_{j}\right| \\
& \geq \frac{1}{\left|\Omega_{j_{n}}\right|} \int_{\Omega_{j_{n}}}\left|v_{j_{n}}\right|^{q_{n}} \mathrm{~d} x \sum_{j=1}^{m}\left|\Omega_{j}\right| \\
& \geq \frac{|\Omega|}{\left|\Omega_{j_{n}}\right|} \int_{\Omega_{j_{n}}}\left|v_{j_{n}}\right|^{q_{n}} \mathrm{~d} x \geq|\Omega|\left(e^{-1-\frac{1}{2}-\frac{1}{3}-\cdots-\frac{1}{N}}\right)^{q_{n}},
\end{aligned}
$$

from which we conclude that

$$
\left(\frac{1}{|\Omega|} \int_{\Omega}|V|^{q_{n}} \mathrm{~d} x\right)^{\frac{1}{q_{n}}} \geq e^{-1-\frac{1}{2}-\frac{1}{3}-\cdots-\frac{1}{N}}>0 .
$$

It follows that

$$
\theta:=\lim _{q \rightarrow 0^{+}}\left(\frac{1}{|\Omega|} \int_{\Omega}|V|^{q} \mathrm{~d} x\right)^{\frac{1}{q}} \geq e^{-1-\frac{1}{2}-\frac{1}{3}-\cdots-\frac{1}{N}}>0 .
$$


Therefore, the function $v:=\theta^{-1} V$ belongs to $W_{0}^{1, p}(\Omega)$, is positive in $\Omega$ and satisfies

$$
\lim _{q \rightarrow 0^{+}}\left(\frac{1}{|\Omega|} \int_{\Omega}|v|^{q} \mathrm{~d} x\right)^{\frac{1}{q}}=1 .
$$

Now, (28) follows immediately since

$$
\mu(\Omega)=\lim _{q \rightarrow 0^{+}} \lambda_{q}(\Omega)|\Omega|^{\frac{p}{q}} \leq \lim _{q \rightarrow 0^{+}} \frac{\|\nabla v\|_{p}^{p}}{\left(\int_{\Omega}|v|^{q} \mathrm{~d} x\right)^{\frac{p}{q}}}|\Omega|^{\frac{p}{q}}=\|\nabla v\|_{p}^{p} .
$$

It might be interesting to know an explicit lower bound for an abstract minimum such as $\mu(\Omega)$. Thus, by combining (24) with (16) we have

$$
\lambda_{1}\left(\Omega^{*}\right)|\Omega|^{p} \leq \lambda_{1}(\Omega)|\Omega|^{p} \leq \mu(\Omega),
$$

where $\Omega^{*}$ denotes the ball centered at the origin with radius $R=\left(|\Omega| / \omega_{N}\right)^{\frac{1}{N}}$, so that $\left|\Omega^{*}\right|=|\Omega|$. It is a known fact (see [5]) that $\lambda_{1}(D)=\left\|\phi_{p, D}\right\|_{1}^{1-p}$, where $D$ is a bounded domain and $\phi_{p, D}$ denotes its $p$-torsion function. Since the $p$-torsion function of a ball $B_{R}$ of radius $R$ is explicitly given by

$$
\phi_{p, B_{R}}(x)=\frac{p-1}{p} N^{-\frac{1}{p-1}}\left(R^{\frac{p}{p-1}}-|x|^{\frac{p}{p-1}}\right), \quad 0 \leq|x| \leq R,
$$

we can compute $\lambda_{1}\left(\Omega^{*}\right)$ explicitly and so obtain, from (29), the following estimate

$$
N\left(N+\frac{p}{p-1}\right)^{p-1}\left(\omega_{N}\right)^{\frac{p}{N}}|\Omega|^{1-\frac{p}{N}} \leq \mu(\Omega) .
$$

For the sake of clarity, we will make use of the following scaling property in the next proof:

$$
\lambda_{q}(\Omega)|\Omega|^{\frac{p}{q}}=|\Omega|^{1-\frac{N}{p}} \lambda_{q}\left(\Omega_{1}\right)
$$

where $\Omega_{1}:=\left\{|\Omega|^{-\frac{1}{N}} x: x \in \Omega\right\}$ is such that $\left|\Omega_{1}\right|=1$. Thus,

$$
\mu(\Omega)=|\Omega|^{1-\frac{N}{p}} \mu\left(\Omega_{1}\right) .
$$

Let us define,

$$
\mathcal{M}(\Omega):=\left\{v \in W_{0}^{1, p}(\Omega): \lim _{q \rightarrow 0^{+}}\left(\frac{1}{|\Omega|} \int_{\Omega}|v|^{q} \mathrm{~d} x\right)^{\frac{1}{q}}=1\right\} .
$$

It is easy to check that $\mathcal{M}(\Omega)$ has infinitely many elements by combining Lemma 6 with the construction in the proof of Theorem 8 .

As pointed out in the Introduction, for each $q \in(0,1)$ there exist $\alpha_{q} \in(0,1)$ and $u_{q} \in W_{0}^{1, p}(\Omega) \cap C^{1, \alpha_{q}}(\bar{\Omega})$ such that

$$
u_{q}>0 \quad \text { in } \quad \Omega, \quad \lambda_{q}(\Omega)=\left\|\nabla u_{q}\right\|_{p}^{p}, \quad \int_{\Omega}\left|u_{q}\right|^{q} \mathrm{~d} x=1
$$

and

$$
\int_{\Omega}\left|\nabla u_{q}\right|^{p-2} \nabla u_{q} \cdot \nabla \varphi \mathrm{d} x=\lambda_{q}(\Omega) \int_{\Omega} u_{q}^{q-1} \varphi \mathrm{d} x \quad \text { for all } \varphi \in W_{0}^{1, p}(\Omega) .
$$

The existence of $u_{q}$ satisfying (32) and (33) is proved in [1], whereas the Hölder regularity of $u_{q}$ follows directly from [10, Theorem 2.2 (i)]. Let us observe that the proof of (33) made in [1] is restricted to the functions $\varphi \in W_{0}^{1, p}(\Omega)$ such that $\operatorname{supp} \varphi \subset \Omega$. However, this restriction can be dropped by using arguments of [9, 14] based on Fatou's lemma combined with the density of $C_{c}^{\infty}(\Omega)$ in $W_{0}^{1, p}(\Omega)$. We will make use of these arguments in the next proof. 
Theorem 9 For each $q \in(0,1)$, let $u_{q} \in W_{0}^{1, p}(\Omega) \cap C^{1, \alpha_{q}}(\bar{\Omega})$ satisfying (32) and (33). There exists $u \in$ $\mathcal{M}(\Omega) \cap C^{0, \alpha}(\bar{\Omega})$, for some $\alpha \in(0,1)$, such that:

(a) $u=\lim _{q \rightarrow 0^{+}}\left(|\Omega|^{\frac{1}{q}} u_{q}\right)$ in $W_{0}^{1, p}(\Omega)$;

(b) $\mu(\Omega)=\|\nabla u\|_{p}^{p}=\min \left\{\|\nabla v\|_{p}^{p}: v \in \mathcal{M}(\Omega)\right\}$;

(c) $-\Delta_{p} u=\frac{\mu(\Omega)}{|\Omega|} u^{-1}, \quad$ in $\Omega$;

(d) $0<A \mu(\Omega)^{\frac{1}{p}} \phi_{p}(x) \leq u(x) \leq B \mu(\Omega)^{\frac{1}{p}}$, for almost every $x \in \Omega$, where $A$ and $B$ are positive constants depending only on $N, p$ and $|\Omega|$.

Proof. Taking (31) into account, we assume in this proof, without loss of generality, that $|\Omega|=1$. Thus,

$$
\lim _{q \rightarrow 0^{+}} \lambda_{q}(\Omega)=\lim _{q \rightarrow 0^{+}}\left\|\nabla u_{q}\right\|_{p}^{p}=\mu(\Omega) \in(0, \infty) .
$$

Since

$$
\lambda_{q}(\Omega)\left(\int_{\Omega}|v|^{q} \mathrm{~d} x\right)^{\frac{p}{q}} \leq\|\nabla v\|_{p}^{p} \quad \text { for all } v \in W_{0}^{1, p}(\Omega)
$$

we have

$$
\mu(\Omega) \leq\|\nabla v\|_{p}^{p} \quad \text { for all } v \in \mathcal{M}(\Omega) .
$$

It follows from (34) that there exist $q_{n} \rightarrow 0^{+}$and $u \in W_{0}^{1, p}(\Omega)$ such that $u \geq 0$ in $\Omega, u_{q_{n}} \rightarrow u$ (weakly) in $W_{0}^{1, p}(\Omega)$ and $u_{q_{n}} \rightarrow u$ pointwise almost everywhere in $\Omega$. Hence,

$$
\|\nabla u\|_{p} \leq \liminf _{n \rightarrow \infty}\left\|\nabla u_{q_{n}}\right\|_{p}=\lim _{n \rightarrow \infty}\left\|\nabla u_{q_{n}}\right\|_{p}=\lim _{n \rightarrow \infty} \lambda_{q_{n}}(\Omega)^{\frac{1}{p}}=\mu(\Omega)^{\frac{1}{p}} .
$$

We note from (35), with $v=u$, that

$$
\mu(\Omega) \lim _{q \rightarrow 0^{+}}\left(\int_{\Omega}|u|^{q} \mathrm{~d} x\right)^{\frac{p}{q}} \leq\|\nabla u\|_{p}^{p} .
$$

Combining this estimate with (37) we obtain

$$
\lim _{q \rightarrow 0^{+}}\left(\int_{\Omega}|u|^{q} \mathrm{~d} x\right)^{\frac{1}{q}} \leq 1 .
$$

On the other hand, for each $s \in(0,1)$ and every $n$ large enough (such that $q_{n}<s$ ), we have

$$
1=\left(\int_{\Omega}\left|u_{q_{n}}\right|^{q_{n}} \mathrm{~d} x\right)^{\frac{1}{q_{n}}} \leq\left(\int_{\Omega}\left|u_{q_{n}}\right|^{s} \mathrm{~d} x\right)^{\frac{1}{s}} .
$$

Hence,

$$
1 \leq \lim _{n \rightarrow \infty}\left(\int_{\Omega}\left|u_{q_{n}}\right|^{s} \mathrm{~d} x\right)^{\frac{1}{s}}=\left(\int_{\Omega}|u|^{s} \mathrm{~d} x\right)^{\frac{1}{s}},
$$

where we have used Dominated Convergence Theorem, since

$$
0 \leq u_{q_{n}} \leq K_{N, p} \lambda_{q_{n}}(\Omega)^{\frac{1}{p-q_{n}}} \leq K_{N, p} \mu(\Omega)^{\frac{1}{p-q_{n}}}
$$

according Lemma 2. Thus, we conclude that

$$
1 \leq \lim _{s \rightarrow 0^{+}}\left(\int_{\Omega}|u|^{s} \mathrm{~d} x\right)^{\frac{1}{s}} .
$$


Gathering (38) and (39) we obtain

$$
\lim _{q \rightarrow 0^{+}}\left(\int_{\Omega}|u|^{q} \mathrm{~d} x\right)^{\frac{1}{q}}=1 .
$$

It follows that $u \in \mathcal{M}(\Omega)$ and thus, by combining (36) and (37) we conclude that

$$
\mu(\Omega)^{\frac{1}{p}}=\|\nabla u\|_{p}=\lim _{n \rightarrow \infty}\left\|\nabla u_{q_{n}}\right\|_{p}
$$

which ends the proof of the claim (b).

Taking into account the weak convergence $u_{q_{n}} \rightarrow u$, the second equality in (40) implies that $u_{q_{n}} \rightarrow u$ (strongly) in $W_{0}^{1, p}(\Omega)$. In view of (33) we have

$$
\int_{\Omega}\left|\nabla u_{q_{n}}\right|^{p-2} \nabla u_{q_{n}} \cdot \nabla \varphi \mathrm{d} x=\lambda_{q_{n}}(\Omega) \int_{\Omega} u_{q_{n}}^{q_{n}-1} \varphi \mathrm{d} x, \quad \text { for all } \varphi \in W_{0}^{1, p}(\Omega) .
$$

Strong convergence $u_{q_{n}} \rightarrow u$ in $W_{0}^{1, p}(\Omega)$ guarantees that

$$
\lim _{n \rightarrow \infty} \int_{\Omega}\left|\nabla u_{q_{n}}\right|^{p-2} \nabla u_{q_{n}} \cdot \nabla \varphi \mathrm{d} x=\int_{\Omega}|\nabla u|^{p-2} \nabla u \cdot \nabla \varphi \mathrm{d} x, \quad \text { for all } \varphi \in W_{0}^{1, p}(\Omega)
$$

and (34) guarantees that

$$
\lim _{n \rightarrow \infty} \lambda_{q_{n}}(\Omega) \int_{\Omega} u_{q_{n}}^{q_{n}-1} \varphi \mathrm{d} x=\mu(\Omega) \lim _{n \rightarrow \infty} \int_{\Omega} u_{q_{n}}^{q_{n}-1} \varphi \mathrm{d} x, \quad \text { for all } \varphi \in W_{0}^{1, p}(\Omega) .
$$

Let us first assume that supp $\varphi \subset \Omega$. Then, Dominated Convergence Theorem yields

$$
\lim _{n \rightarrow \infty} \int_{\Omega} u_{q_{n}}^{q_{n}-1} \varphi \mathrm{d} x=\int_{\Omega} u^{-1} \varphi \mathrm{d} x
$$

since Lemma 2 and Lemma 3 imply that $0<c_{1} \leq u_{q_{n}}^{q_{n}-1} \varphi \leq c_{2}$ in $\operatorname{supp} \varphi$, where the constants $c_{1}$ and $c_{2}$ are uniform with respect to $n$. Hence, by gathering (41), (42) and (43) we have

$$
\int_{\Omega}|\nabla u|^{p-2} \nabla u \cdot \nabla \varphi \mathrm{d} x=\mu(\Omega) \int_{\Omega} \varphi u^{-1} \mathrm{~d} x, \quad \text { for all } \varphi \in C_{c}^{\infty}(\Omega) .
$$

Thus, in order to prove (c) we need to show that (44) holds, in fact, for any $\varphi \in W_{0}^{1, p}(\Omega)$, which reduces to prove that (43) holds for any $\varphi \in W_{0}^{1, p}(\Omega)$. We prove this by following arguments of [9, 14. So, let $w \in W_{0}^{1, p}(\Omega)$ be arbitrary and take a sequence $\left\{\xi_{n}\right\} \subset C_{c}^{\infty}(\Omega)$ of nonnegative functions such that $\xi_{n} \rightarrow|w|$, strongly in $W_{0}^{1, p}(\Omega)$ and pointwise almost everywhere in $\Omega$. Hence, by applying: Fatou's lemma, (44) and Hölder's inequality, we obtain

$$
\begin{aligned}
\left|\int_{\Omega} w u^{-1} \mathrm{~d} x\right| & \leq \int_{\Omega}|w| u^{-1} \mathrm{~d} x \\
& \leq \liminf _{n \rightarrow \infty} \int_{\Omega} \xi_{n} u^{-1} \mathrm{~d} x \\
& =\lim _{n \rightarrow \infty} \int_{\Omega}|\nabla u|^{p-2} \nabla u \cdot \nabla \xi_{n} \mathrm{~d} x \leq\|\nabla u\|_{p}^{p-1} \lim _{n \rightarrow \infty}\left\|\nabla \xi_{n}\right\|_{p}=\|\nabla u\|_{p}^{p-1}\|\nabla w\|_{p} .
\end{aligned}
$$

Now, let $\varphi$ be an arbitrary function in $W_{0}^{1, p}(\Omega)$ and take $\left\{\varphi_{n}\right\} \subset C_{c}^{\infty}(\Omega)$ such that $\varphi_{n} \rightarrow \varphi$ strongly in $W_{0}^{1, p}(\Omega)$. Then, by using $\varphi_{n}-\varphi$ in the place of $w$, we obtain

$$
\lim _{n \rightarrow \infty}\left|\int_{\Omega}\left(\varphi_{n}-\varphi\right) u^{-1} \mathrm{~d} x\right| \leq\|\nabla u\|_{p}^{p-1} \lim _{n \rightarrow \infty}\left\|\nabla\left(\varphi_{n}-\varphi\right)\right\|_{p}=0
$$


which yields

$$
\lim _{n \rightarrow \infty} \int_{\Omega} \varphi_{n} u^{-1} \mathrm{~d} x=\int_{\Omega} \varphi u^{-1} \mathrm{~d} x .
$$

Since $\varphi_{n} \in C_{c}^{\infty}(\Omega)$ we obtain from (44) that

$$
\lim _{n \rightarrow \infty} \int_{\Omega} \varphi_{n} u^{-1} \mathrm{~d} x=\mu(\Omega)^{-1} \lim _{n \rightarrow \infty} \int_{\Omega}|\nabla u|^{p-2} \nabla u \cdot \nabla \varphi_{n} \mathrm{~d} x=\mu(\Omega)^{-1} \int_{\Omega}|\nabla u|^{p-2} \nabla u \cdot \nabla \varphi \mathrm{d} x .
$$

Therefore, by combining (45) with (46) we conclude that (43) holds true for any $\varphi \in W_{0}^{1, p}(\Omega)$, which proves the claim (c).

Claim (d) now follows after combining Lemma 2 with Lemma 3 Theorem 2.2 (ii) of [10] implies that $u \in$ $C^{0, \alpha}(\bar{\Omega})$ for some $\alpha \in(0,1)$.

Claim (a) follows from the uniqueness of the weak solutions of

$$
\begin{cases}-\Delta_{p} w=\frac{\mu(\Omega)}{|\Omega|} w^{-1} & \text { in } \Omega, \\ w>0 & \text { in } \Omega, \\ w=0 & \text { on } \partial \Omega\end{cases}
$$

combined with the fact that $u_{q_{n}} \rightarrow u$ strongly in $W_{0}^{1, p}(\Omega)$. Indeed, these facts together imply that $u$ is the unique limit function of the family $\left\{u_{q}\right\}$, as $q \rightarrow 0^{+}$.

Our next goal is to prove that the solution $u$ of (47) satisfies

$$
\int_{\Omega} \log u \mathrm{~d} x=0 .
$$

Proposition 10 Let $v \in L^{1}(\Omega)$. Then $\log |v|$ is Lebesgue measurable in $\Omega$ and

$$
-\infty \leq \lim _{q \rightarrow 0^{+}} \int_{\Omega}|v|^{q} \log |v| \mathrm{d} x=\int_{\Omega} \log |v| \mathrm{d} x \leq 2 e^{-1}\|v\|_{1} .
$$

Proof. For every $x \in \Omega$ such that $|v(x)| \leq 1$ the function $q \in(0,1] \mapsto-|v(x)|^{q} \log |v(x)| \in[0, \infty]$ is decreasing and

$$
\lim _{q \rightarrow 0^{+}}\left[-|v(x)|^{q} \log |v(x)|\right]=-\log |v(x)| .
$$

Therefore, it follows directly from Lebesgue's Monotone Convergence Theorem that $\log |v|$ is Lebesgue measurable in the set $\{x \in \Omega:|v(x)| \leq 1\}$ and

$$
-\infty \leq \lim _{q \rightarrow 0^{+}} \int_{\{|v| \leq 1\}}|v|^{q} \log |v| \mathrm{d} x=\int_{\{|v| \leq 1\}} \log |v| \mathrm{d} x \leq 0 .
$$

Now, for every $x \in \Omega$ such that $|v(x)| \geq 1$ the function $q \in(0,1] \mapsto|v(x)|^{q} \log |v(x)| \in[0, \infty]$ is increasing and

$$
\lim _{q \rightarrow 0^{+}}|v(x)|^{q} \log |v(x)|=\log |v(x)| .
$$

Moreover, for every $q \in\left(0, \frac{1}{2}\right)$ one has

$$
0 \leq|v(x)|^{q} \log |v(x)| \leq|v(x)|^{\frac{1}{2}} \log |v(x)| \leq 2 e^{-1}|v(x)|^{\frac{1}{2}}|v(x)|^{\frac{1}{2}}=2 e^{-1}|v(x)| \in L^{1}(\Omega),
$$

since $\max _{t \geq 1} t^{-\frac{1}{2}} \log t=2 e^{-1}$. Therefore, Lebesgue's Dominated Convergence Theorem implies that $\log |v|$ is integrable in the set $\{x \in \Omega:|v(x)| \geq 1\}$ and that

$$
0 \leq \lim _{q \rightarrow 0^{+}} \int_{\{|v| \geq 1\}}|v|^{q} \log |v| \mathrm{d} x=\int_{\{|v| \geq 1\}} \log |v| \mathrm{d} x \leq 2 e^{-1}\|v\|_{1} .
$$


Thus, we have that $\log |v|$ is Lebesgue measurable in $\Omega$ and

$$
\begin{aligned}
\lim _{q \rightarrow 0^{+}} \int_{\Omega}|v|^{q} \log |v| \mathrm{d} x & =\lim _{q \rightarrow 0^{+}} \int_{\{|v| \leq 1\}}|v|^{q} \log |v| \mathrm{d} x+\lim _{q \rightarrow 0^{+}} \int_{\{|v|>1\}}|v|^{q} \log |v| \mathrm{d} x \\
& =\int_{\{|v| \leq 1\}} \log |v| \mathrm{d} x+\int_{\{|v|>1\}} \log |v| \mathrm{d} x \\
& =\int_{\Omega} \log |v| \mathrm{d} x \leq 2 e^{-1}\|v\|_{1} .
\end{aligned}
$$

Proposition 11 For $v \in L^{1}(\Omega)$ define

$$
\theta_{v}:=\lim _{q \rightarrow 0^{+}}\left(\frac{1}{|\Omega|} \int_{\Omega}|v|^{q} \mathrm{~d} x\right)^{\frac{1}{q}} \in[0, \infty) \text { and } \beta_{v}:=\frac{1}{|\Omega|} \int_{\Omega} \log |v| \mathrm{d} x \in[-\infty, \infty) .
$$

Then

$$
\beta_{v}=\log \theta_{v}
$$

Proof. It follows from L'Hôpital's rule and Proposition 10 that

$$
\lim _{q \rightarrow 0^{+}}\left(\frac{1}{|\Omega|} \int_{\Omega}|v|^{q} \mathrm{~d} x\right)^{\frac{1}{q}}=\exp \left(\frac{1}{|\Omega|} \lim _{q \rightarrow 0^{+}} \int_{\Omega}|v|^{q} \log |v| \mathrm{d} x\right)=\exp \left(\frac{1}{|\Omega|} \int_{\Omega} \log |v| \mathrm{d} x\right) .
$$

Hence, (48) follows.

The following corollary is an immediate consequence of the previous proposition.

Corollary 12 Let $v \in W_{0}^{1, p}(\Omega)$. Then $v \in \mathcal{M}(\Omega)$ if, and only if,

$$
\int_{\Omega} \log |v| \mathrm{d} x=0
$$

In particular, $\int_{\Omega} \log |u| \mathrm{d} x=0$, where $u$ is the solution of (47).

\subsection{Minimizing the energy functional}

In this subsection, $u$ denotes the solution of (47). As we have shown, $u$ minimizes the functional $v \mapsto\|\nabla v\|_{p}^{p}$ on $\mathcal{M}(\Omega)$. Let us show that $u$ is the unique, up to sign, with this property.

Lemma 13 Let $v \in \mathcal{M}(\Omega)$ such that $\mu(\Omega)=\|\nabla v\|_{p}^{p}$. Then, $v$ does not change sign in $\Omega$.

Proof. Let $\Omega_{+}:=\{x \in \Omega: v(x)>0\}, \Omega_{-}=\{x \in \Omega: v(x)<0\}, a_{+}:=\frac{\left|\Omega_{+}\right|}{|\Omega|}$ and $a_{-}:=\frac{\left|\Omega_{-}\right|}{|\Omega|}$. For $0<q<1$, we have

$$
\begin{aligned}
\left(\frac{1}{|\Omega|} \int_{\Omega}|v|^{q} \mathrm{~d} x\right)^{\frac{1}{q}} & =\left(\frac{a_{+}}{\left|\Omega_{+}\right|} \int_{\Omega_{+}}\left|v_{+}\right|^{q} \mathrm{~d} x+\frac{a_{-}}{\left|\Omega_{-}\right|} \int_{\Omega_{-}}\left|v_{-}\right|^{q} \mathrm{~d} x\right)^{\frac{1}{q}} \\
& \leq a_{+}\left(\frac{1}{\left|\Omega_{+}\right|} \int_{\Omega_{+}}\left|v_{+}\right|^{q} \mathrm{~d} x\right)^{\frac{1}{q}}+a_{-}\left(\frac{1}{\left|\Omega_{-}\right|} \int_{\Omega_{-}}\left|v_{-}\right|^{q} \mathrm{~d} x\right)^{\frac{1}{q}} .
\end{aligned}
$$

It follows that

$$
\begin{aligned}
1 & =\lim _{q \rightarrow 0^{+}}\left(\frac{1}{|\Omega|} \int_{\Omega}|v|^{q} \mathrm{~d} x\right)^{\frac{1}{q}} \\
& \leq\left(a_{+}\right) \lim _{q \rightarrow 0^{+}}\left(\frac{1}{\left|\Omega_{+}\right|} \int_{\Omega_{+}}\left|v_{+}\right|^{q} \mathrm{~d} x\right)^{\frac{1}{q}}+\left(a_{-}\right) \lim _{q \rightarrow 0^{+}}\left(\frac{1}{\left|\Omega_{-}\right|} \int_{\Omega_{-}}\left|v_{-}\right|^{q} \mathrm{~d} x\right)^{\frac{1}{q}} .
\end{aligned}
$$



that

Let us suppose, by contradiction, that both $\left|\Omega_{+}\right|$and $\left|\Omega_{-}\right|$are positive. Without loss of generality, we assume

$$
\lim _{q \rightarrow 0^{+}}\left(\frac{1}{\left|\Omega_{-}\right|} \int_{\Omega_{-}}\left|v_{-}\right|^{q} \mathrm{~d} x\right)^{\frac{1}{q}} \leq \theta_{+}:=\lim _{q \rightarrow 0^{+}}\left(\frac{1}{\left|\Omega_{+}\right|} \int_{\Omega_{+}}\left|v_{+}\right|^{q} \mathrm{~d} x\right)^{\frac{1}{q}} .
$$

Then

$$
1 \leq\left(a_{+}+a_{-}\right) \lim _{q \rightarrow 0^{+}}\left(\frac{1}{\left|\Omega_{+}\right|} \int_{\Omega_{+}}\left|v_{+}\right|^{q} \mathrm{~d} x\right)^{\frac{1}{q}}=\theta_{+} .
$$

It follows that $\theta_{+}^{-1} v_{+} \in \mathcal{M}(\Omega)$ and

$$
\mu(\Omega) \leq\left\|\nabla\left(\theta_{+}^{-1} v_{+}\right)\right\|_{p}^{p} \leq\left\|\nabla\left(v_{+}\right)\right\|_{p}^{p} \leq\|\nabla v\|_{p}^{p}=\mu(\Omega)
$$

Thus,

$$
\mu(\Omega)=\left\|\nabla v_{+}\right\|_{p}^{p}=\|\nabla v\|_{p}^{p}=\left\|\nabla v_{+}\right\|_{p}^{p}+\left\|\nabla v_{-}\right\|_{p}^{p}
$$

implying that $\left\|\nabla v_{-}\right\|_{p}^{p}=0$ and, therefore, that $v_{-}=0$ a.e. in $\Omega$. This implies that $\left|\Omega_{-}\right|=0$, which is a contradiction.

Theorem 14 Let $v \in \mathcal{M}(\Omega)$ such that $\mu(\Omega)=\|\nabla v\|_{p}^{p}$. Then, $v= \pm u$ a.e. in $\Omega$.

Proof. According Lemma 13, we can assume, without loss of generality, that $v \geq 0$ a. e. in $\Omega$. Since $0<q<1$, it is simple to check that

$$
\left(\int_{\Omega}\left(\frac{u+v}{2}\right)^{q} \mathrm{~d} x\right)^{\frac{1}{q}} \geq\left(\int_{\Omega}\left(\frac{u}{2}\right)^{q} \mathrm{~d} x\right)^{\frac{1}{q}}+\left(\int_{\Omega}\left(\frac{v}{2}\right)^{q} \mathrm{~d} x\right)^{\frac{1}{q}}
$$

Thus,

$$
h:=\lim _{q \rightarrow 0^{+}}\left(\frac{1}{|\Omega|} \int_{\Omega}\left(\frac{u+v}{2}\right)^{q} \mathrm{~d} x\right)^{\frac{1}{q}} \geq \frac{1}{2}+\frac{1}{2}=1 .
$$

Of course, $\frac{u+v}{2 h} \in \mathcal{M}(\Omega)$. Thus,

$$
\mu(\Omega) \leq\left\|\nabla\left(\frac{u+v}{2 h}\right)\right\|_{p}^{p} \leq \frac{1}{(2 h)^{p}}\left(\|\nabla u\|_{p}+\|\nabla v\|_{p}\right)^{p}=\frac{\left(2 \mu(\Omega)^{\frac{1}{p}}\right)^{p}}{(2 h)^{p}}=\frac{\mu(\Omega)}{h^{p}},
$$

which implies that $h \leq 1$. Therefore, (49) and (50) imply that $h=1$, and this fact in (50) yields

$$
\|\nabla(u+v)\|_{p}=\|\nabla u\|_{p}+\|\nabla v\|_{p} .
$$

We conclude from this equality that $u=v$ a. e. in $\Omega$.

The next corollary shows that $\mu(\Omega)^{-1}$ is the best constant $C$ in the following log-Sobolev type inequality

$$
\exp \left(\frac{1}{|\Omega|} \int_{\Omega} \log |v|^{p} \mathrm{~d} x\right) \leq C\|\nabla v\|_{p}^{p}, \quad v \in W_{0}^{1, p}(\Omega)
$$

and that when $C=\mu(\Omega)^{-1}$ this inequality becomes an equality if, and only if, $v$ is a scalar multiple of $u$.

Corollary 15 One has

$$
\mu(\Omega)=\min \left\{\frac{\|\nabla v\|_{p}^{p}}{\exp \left(\frac{1}{|\Omega|} \int_{\Omega} \log |v|^{p} \mathrm{~d} x\right)}: v \in W_{0}^{1, p}(\Omega) \text { and } \int_{\Omega} \log |v|^{p} \mathrm{~d} x>-\infty\right\} .
$$

Moreover, the minimum is reached only by scalar multiples of $u$. 
Proof. Let $v \in W_{0}^{1, p}(\Omega)$ be such that $\int_{\Omega} \log |v|^{p} \mathrm{~d} x>-\infty$. Since

$$
\lambda_{q}(\Omega)|\Omega|^{\frac{p}{q}} \leq \frac{\|\nabla v\|_{p}^{p}}{\left(\frac{1}{|\Omega|} \int_{\Omega}|v|^{q} \mathrm{~d} x\right)^{\frac{p}{q}}}
$$

we obtain

$$
\mu(\Omega)=\lim _{q \rightarrow 0^{+}} \lambda_{q}(\Omega)|\Omega|^{\frac{p}{q}} \leq \frac{\|\nabla v\|_{p}^{p}}{\lim _{q \rightarrow 0^{+}}\left(\frac{1}{|\Omega|} \int_{\Omega}|v|^{q} \mathrm{~d} x\right)^{\frac{p}{q}}}=\frac{\|\nabla v\|_{p}^{p}}{\exp \left(\frac{1}{|\Omega|} \int_{\Omega} \log |v|^{p} \mathrm{~d} x\right)} .
$$

Since $\int_{\Omega} \log |u| \mathrm{d} x=0$, we have

$$
\frac{\|\nabla u\|_{p}^{p}}{\exp \left(\frac{1}{|\Omega|} \int_{\Omega} \log |u|^{p} \mathrm{~d} x\right)}=\|\nabla u\|_{p}^{p}=\mu(\Omega) .
$$

Of course, if $v \in W_{0}^{1, p}(\Omega)$ is such that

$$
\frac{\|\nabla v\|_{p}^{p}}{\exp \left(\frac{1}{|\Omega|} \int_{\Omega} \log |v|^{p} \mathrm{~d} x\right)}=\mu(\Omega)
$$

then $\theta_{v}^{-1} v \in \mathcal{M}(\Omega)$, where

$$
\theta_{v}=\lim _{q \rightarrow 0^{+}}\left(\frac{1}{|\Omega|} \int_{\Omega}|v|^{q} \mathrm{~d} x\right)^{\frac{1}{q}}=\exp \left(\frac{1}{|\Omega|} \int_{\Omega} \log |v|^{p} \mathrm{~d} x\right)>0,
$$

and $\left\|\nabla\left(\theta_{v}^{-1} v\right)\right\|_{p}^{p}=\mu(\Omega)$. Hence, $\theta_{v}^{-1} v= \pm u$, implying that $v$ is a scalar multiple of $u$.

Remark 16 Gathering (30) and (51), with $C=\mu(\Omega)^{-1}$, we obtain

$$
\exp \left(\frac{1}{|\Omega|} \int_{\Omega} \log |v|^{p} \mathrm{~d} x\right) \leq C_{N, p,|\Omega|}\|\nabla v\|_{p}^{p}, \quad v \in W_{0}^{1, p}(\Omega),
$$

where $C_{N, p,|\Omega|}:=N^{-1}\left(N+\frac{p}{p-1}\right)^{1-p}\left(\omega_{N}\right)^{-\frac{p}{N}}|\Omega|^{\frac{p}{N}-1}$.

Now, let us define $J: W_{0}^{1, p}(\Omega) \mapsto(-\infty, \infty]$, the formal energy functional, by

$$
J(v):= \begin{cases}\frac{1}{p} \int_{\Omega}|\nabla v|^{p} \mathrm{~d} x-\frac{\mu(\Omega)}{|\Omega|} \int_{\Omega} \log |v| \mathrm{d} x, & \text { if } \int_{\Omega} \log |v| \mathrm{d} x \in(-\infty, \infty) \\ \infty, & \text { if } \int_{\Omega} \log |v| \mathrm{d} x=-\infty .\end{cases}
$$

We are going to show that $u$ is the unique minimizer of $J$ in $W_{0}^{1, p}(\Omega)$.

\section{Lemma 17 One has}

$$
J(v) \geq \frac{\mu(\Omega)}{p}-\frac{\mu(\Omega)}{p} \log \left(\frac{\mu(\Omega)}{\|\nabla v\|_{p}^{p}}\right)-\frac{\mu(\Omega)}{|\Omega|} \int_{\Omega} \log |v| \mathrm{d} x, \quad \text { for all } v \in W_{0}^{1, p}(\Omega) .
$$

Proof. Let $v \in W_{0}^{1, p}(\Omega)$ and consider the function

$$
g(t):=\frac{t^{p}}{p}\|\nabla v\|_{p}^{p}-\mu(\Omega) \log t-\frac{\mu(\Omega)}{|\Omega|} \int_{\Omega} \log |v| \mathrm{d} x, \quad t>0 .
$$

It is easy to check that $J(t v)=g(|t|)$ and that $\min _{t>0} g(t)$ is the right-hand side of (52). The result then follows, since $J(v)=g(1) \geq \min _{t>0} g(t)$. 
Theorem 18 One has

$$
J(u)=\frac{\mu(\Omega)}{p}=\min _{v \in W_{0}^{1, p}(\Omega)} J(v) .
$$

Proof. Since

$$
\mu(\Omega)=\int_{\Omega}|\nabla u|^{p} \mathrm{~d} x \text { and } \int_{\Omega} \log |u| \mathrm{d} x=0
$$

we have $J(u)=\frac{\mu(\Omega)}{p}$. Thus, we need only to prove that

$$
J(v) \geq \frac{\mu(\Omega)}{p}, \quad \text { for all } v \in W_{0}^{1, p}(\Omega) .
$$

Let $v \in W_{0}^{1, p}(\Omega)$ and $\theta_{v}$ as in Proposition [1]. If $\theta_{v}=0$, then $J(v)=\infty$ and (53) holds trivially. If $\theta_{v}>0$ then $\theta_{v}^{-1} v \in \mathcal{M}(\Omega)$ and

$$
\frac{1}{|\Omega|} \int_{\Omega} \log \left|\theta_{v}^{-1} v\right| \mathrm{d} x=0 \text { and } \mu(\Omega) \leq\left\|\nabla\left(\theta_{v}^{-1} v\right)\right\|_{p}^{p}=\theta_{v}^{-p}\|\nabla v\|_{p}^{p},
$$

implying, respectively, that

$$
\frac{1}{|\Omega|} \int_{\Omega} \log |v| \mathrm{d} x=\log \theta_{v}
$$

and

$$
\begin{aligned}
J(v) & \geq \frac{\mu(\Omega)}{p}-\frac{\mu(\Omega)}{p} \log \left(\frac{\mu(\Omega)}{\|\nabla v\|_{p}^{p}}\right)-\mu(\Omega) \log \left(\theta_{v}\right) \\
& \geq \frac{\mu(\Omega)}{p}-\frac{\mu(\Omega)}{p} \log \left(\theta_{v}^{-p}\right)-\mu(\Omega) \log \left(\theta_{v}\right)=\frac{\mu(\Omega)}{p} .
\end{aligned}
$$

Theorem 19 If $w \in W_{0}^{1, p}(\Omega)$ minimizes $J$, then $w= \pm \theta_{w} u$ a.e. in $\Omega$, where

$$
\theta_{w}:=\lim _{q \rightarrow 0^{+}}\left(\frac{1}{|\Omega|} \int_{\Omega}|w|^{q} \mathrm{~d} x\right)^{\frac{1}{q}}
$$

Proof. It follows from Lemma 17 that

$$
\frac{\mu(\Omega)}{p}=J(w) \geq \frac{\mu(\Omega)}{p}-\frac{\mu(\Omega)}{p} \log \left(\frac{\mu(\Omega)}{\|\nabla w\|_{p}^{p}}\right)-\frac{\mu(\Omega)}{|\Omega|} \int_{\Omega} \log |w| \mathrm{d} x,
$$

which implies that

$$
\frac{1}{p} \log \left(\frac{\mu(\Omega)}{\|\nabla w\|_{p}^{p}}\right)+\frac{1}{|\Omega|} \int_{\Omega} \log |w| \mathrm{d} x \geq 0 .
$$

Of course, $\theta_{w} \in(0, \infty)$, so that $\theta_{w}^{-1} w \in \mathcal{M}(\Omega)$. Thus, according Corollary 12

$$
\frac{1}{|\Omega|} \int_{\Omega} \log \left|\theta_{w}^{-1} w\right| \mathrm{d} x=0
$$

so that

$$
\log \theta_{w}=\frac{1}{|\Omega|} \int_{\Omega} \log |w| \mathrm{d} x
$$


Hence, (54) yields

$$
\frac{1}{p} \log \left(\frac{\mu(\Omega)}{\|\nabla w\|_{p}^{p}}\right)+\log \theta_{w}=\log \left(\frac{\mu(\Omega)}{\left\|\nabla\left(\theta_{w}^{-1} w\right)\right\|_{p}^{p}}\right)^{\frac{1}{p}} \geq 0,
$$

which is equivalent to $\mu(\Omega) \geq\left\|\nabla \theta_{w}^{-1} w\right\|_{p}^{p}$.

Since $\mu(\Omega) \leq\left\|\nabla \theta_{w}^{-1} w\right\|_{p}^{p}$ (recall that $\theta_{w}^{-1} w \in \mathcal{M}(\Omega)$ ), we conclude that $\mu(\Omega)=\left\|\nabla\left(\theta_{w}^{-1} w\right)\right\|_{p}^{p}$. So, Theorem 14 yields $\theta_{w}^{-1} w= \pm u$.

We end this section by remarking that, for each $\lambda>0$, a simple scaling argument shows that the function

$$
u_{\lambda}:=\left(\frac{\lambda|\Omega|}{\mu(\Omega)}\right)^{\frac{1}{p}} u
$$

is the unique solution of the singular problem

$$
\begin{cases}-\Delta_{p} v=\lambda v^{-1} & \text { in } \Omega \\ v>0 & \text { in } \Omega \\ v=0 & \text { on } \partial \Omega .\end{cases}
$$

Moreover, since $\int_{\Omega} \log u \mathrm{~d} x=0$ and $\mu(\Omega)=\|\nabla u\|_{p}^{p}$, the equality in (51) (with $C=\mu(\Omega)^{-1}$ ) yields

$$
\int_{\Omega} \log u_{\lambda} \mathrm{d} x=\frac{|\Omega|}{p} \log \left(\mu(\Omega)^{-1}\left(\frac{\lambda|\Omega|}{\mu(\Omega)}\right)\|\nabla u\|_{p}^{p}\right)=\frac{|\Omega|}{p} \log \left(\frac{\lambda|\Omega|}{\mu(\Omega)}\right) .
$$

We also note that $u_{\lambda}$ and $-u_{\lambda}$ are the unique minimizers of the functional

$$
J_{\lambda}(v):= \begin{cases}\frac{1}{p} \int_{\Omega}|\nabla v|^{p} \mathrm{~d} x-\lambda \int_{\Omega} \log |v| \mathrm{d} x, & \text { if } \int_{\Omega} \log |v| \mathrm{d} x \in(-\infty, \infty) \\ \infty, & \text { if } \int_{\Omega} \log |v| \mathrm{d} x=-\infty,\end{cases}
$$

being

$$
J_{\lambda}\left( \pm u_{\lambda}\right)=\frac{\mu(\Omega)}{p}-\frac{\lambda|\Omega|}{p} \log \left(\frac{\lambda|\Omega|}{\mu(\Omega)}\right) .
$$

\subsection{Asymptotics for the pair $\left(\lambda_{q}(\Omega),\left\|u_{q}\right\|_{\infty}\right)$}

In this subsection we describe the asymptotic behavior of $\lambda_{q}(\Omega)$, as $q \rightarrow 0^{+}$. Of course, if $|\Omega|=1$, then $\lim _{q \rightarrow 0^{+}} \lambda_{q}(\Omega)=\mu(\Omega)$ and, according items (a) and (d) of Theorem 9

$$
0<A \mu(\Omega)^{\frac{1}{p}}\left\|\phi_{p}\right\|_{\infty} \leq \lim _{q \rightarrow 0^{+}}\left\|u_{q}\right\|_{\infty} \leq B \mu(\Omega)^{\frac{1}{p}}
$$

where $A$ and $B$ are positive constants depending only on $N$ and $p$. If $|\Omega| \neq 1$ we have

$$
\lim _{q \rightarrow 0^{+}} \lambda_{q}(\Omega)=\lim _{q \rightarrow 0^{+}}\left(\lambda_{q}(\Omega)|\Omega|^{\frac{p}{q}}\right)\left(\lim _{q \rightarrow 0^{+}}|\Omega|^{-\frac{p}{q}}\right)=\mu(\Omega)\left(\lim _{q \rightarrow 0^{+}}|\Omega|^{-\frac{p}{q}}\right) .
$$

Therefore, in this case, we readily obtain

$$
\lim _{q \rightarrow 0^{+}} \lambda_{q}(\Omega)=\left\{\begin{array}{lll}
\infty & \text { if } & |\Omega|<1 \\
0 & \text { if } & |\Omega|>1 .
\end{array}\right.
$$

Hence, by combining (55) with Lemma 2 and Lemma 3 we conclude that

$$
\lim _{q \rightarrow 0^{+}}\left\|u_{q}\right\|_{\infty}=\left\{\begin{array}{lll}
\infty & \text { if } & |\Omega|<1 \\
0 & \text { if } & |\Omega|>1 .
\end{array}\right.
$$


We remark that (55) is also simple to prove without using Theorem 9 In fact, in the case $|\Omega|<1$ the monotonicity of the function $q \mapsto \lambda_{q}(\Omega)|\Omega|^{\frac{p}{q}}$ implies that

$$
\lim _{q \rightarrow 0^{+}} \lambda_{q}(\Omega)=\lim _{q \rightarrow 0^{+}} \lambda_{q}(\Omega)|\Omega|^{\frac{p}{q}}|\Omega|^{-\frac{p}{q}} \geq \lambda_{1}(\Omega)|\Omega|^{p} \lim _{q \rightarrow 0^{+}}|\Omega|^{-\frac{p}{q}}=\infty .
$$

This is the proof given in [1]. As for the case $|\Omega|>1$, take $v \in W_{0}^{1, p}(\Omega) \cap C(\bar{\Omega})$ such that $v>0$ in $\Omega$ and then define $\Omega_{\epsilon}:=\{x \in \Omega: v(x) \geq \epsilon\}$, where $\epsilon>0$ is such that $\left|\Omega_{\epsilon}\right|>1$.Then,

$$
0<\lambda_{q}(\Omega) \leq \frac{\int_{\Omega}|\nabla v|^{p} \mathrm{~d} x}{\left(\int_{\Omega}|v|^{q} \mathrm{~d} x\right)^{\frac{p}{q}}} \leq \frac{\int_{\Omega}|\nabla v|^{p} \mathrm{~d} x}{\left(\int_{\Omega_{\epsilon}} \epsilon^{q} \mathrm{~d} x\right)^{\frac{p}{q}}}=\frac{\int_{\Omega}|\nabla v|^{p} \mathrm{~d} x}{\epsilon^{p}\left|\Omega_{\epsilon}\right|^{\frac{p}{q}}} .
$$

Thus, by making $q \rightarrow 0^{+}$, we obtain $\lim _{q \rightarrow 0^{+}} \lambda_{q}(\Omega)=0$, since $\lim _{q \rightarrow 0^{+}}\left|\Omega_{\epsilon}\right|^{-\frac{p}{q}}=0$.

\section{Acknowledgments}

The first author thanks the support of Fundação de Amparo à Pesquisa do Estado de Minas Gerais (Fapemig)/Brazil (CEX-PPM-00165) and Conselho Nacional de Desenvolvimento Científico e Tecnológico (CNPq)/Brazil (483970/20131 and 306590/2014-0).

\section{References}

[1] G. Anello, F. Faraci and A. Iannizzotto: On a problem of Huang concerning best constants in Sobolev embeddings, Ann. Mat. Pura Appl. 194 (2015) 767-779.

[2] C. Bandle: Rayleigh-Faber-Krahn inequalities and quasilinear elliptic boundary value problems, Nonlinear analysis and applications: to V. Lakshmikantham on his 80th birthday. Vol. 1,2, Kluwer Acad. Publ., Dordrecht, 2003, p.p 227-240.

[3] Y. Chu and W. Gao: Existence of solutions to a class of quasilinear elliptic problems with nonlinear singular terms, Boundary Value Problems 2013:229 (2013).

[4] M. G. Crandall, P. H. Rabinowitz and L. Tartar: On a Dirichlet problem with singular nonlinearity, Comm. Partial Differential Equations 2 (1977) 193-222.

[5] G. Ercole: Absolute continuity of the best Sobolev constant, J. Math. Anal. Appl. 404 (2013) 420-428.

[6] G. Ercole: On the resonant Lane-Emden problem for the p-Laplacian, Comm. Contemp. Math. 16 (2014) 1350023 (22 pages).

[7] G. Ercole and G. A. Pereira: Asymptotics for the best Sobolev constants and their extremal functions, Math. Nachr. (to appear) DOI 10.1002/mana.201500263.

[8] G. P. Galdi: An Introduction to the Mathematical Theory of the Navier-Stokes Equations, Springer, New York, 2011.

[9] J. Giacomoni, I. Schindler and P. Takáč: Sobolev versus Hölder local minimizers and existence of multiple solutions for a singular quasilinear equation, Ann. Scuola Norm. Sup. Pisa Cl. Sci. 6 (2007) 117-158.

[10] J. Giacomoni, I. Schindler and P. Takáč: Singular quasilinear elliptic equations and Hölder regularity, C. R. Acad. Sci. Paris, Ser. I 350 (2012) 383-388.

[11] B. Kawohl: Rearrangements and Convexity of Level Sets in PDE, Lecture Notes in Math., vol. 1150, SpringerVerlag, Berlin, 1985. 
[12] O. Ladyzhenskaya and N. Ural'tseva: Linear and Quasilinear Elliptic Equations, Academic Press, New YorkLondon, 1968.

[13] A. C. Lazer and P. J. Mckenna: On a singular nonlinear elliptic boundary value problem. Proc. Am. Math. Soc. 111 (1991) 721-730.

[14] A. Mohammed: Positive solutions of the p-Laplace equation with singular nonlinearity, J. Math. Anal. Appl. 352 (2009) 234-245.

[15] X. Ren and J. Wei: Counting peaks of solutions to some quasilinear elliptic equations with large exponents, J. Differential Equations 117 (1995) 28-55.

[16] C. A. Stuart: Existence and approximation of solutions of nonlinear elliptic equations, Math. Z. 147 (1976) 53-63. 